\title{
Effects of Empathy and Conflict Resolution Strategies on Psychophysiological Arousal and Satisfaction in Romantic Relationships
}

\author{
Kristin M. Perrone-McGovern • Patrícia Oliveira-Silva • \\ Stephanie Simon-Dack • Erin Lefdahl-Davis • \\ David Adams • John McConnell • Desiree Howell • \\ Ryan Hess · Andrew Davis • Óscar F. Gonçalves
}

Published online: 9 November 2013

(C) Springer Science+Business Media New York 2013

\begin{abstract}
The present research builds upon the extant literature as it assesses psychophysiological factors in relation to empathy, conflict resolution, and romantic relationship satisfaction. In this study, we examined physiological reactivity of individuals in the context of emotionally laden interactions with their romantic partners. Participants $(\mathrm{N}=31)$ completed self-report measures and attended in-person data collection sessions with their romantic partners. Participants were guided through discussions of problems and strengths of their relationships in vivo with their partners while we measured participants' skin conductance level (SCL) and interbeat interval (IBI) of the heart. We hypothesized that participants' level of empathy towards their partners would be reflected by physiological arousal (as measured by SCL and IBI) and relationship satisfaction, such that higher levels of empathy would be linked to changes in physiological arousal and higher relationship satisfaction. Further, we hypothesized that differences would be found in physiological arousal (as measured by SCL and IBI) based on the type of conflict resolution strategy used by participants. Finally, we hypothesized that differences would be found in empathy towards partner and relationship satisfaction based on the type of conflict resolution strategies used by participants.
\end{abstract}

K. M. Perrone-McGovern $(\varangle) \cdot$ S. Simon-Dack .

E. Lefdahl-Davis - D. Adams - J. McConnell - D. Howell ·

R. Hess - A. Davis

Ball State University, Muncie, IN, USA

e-mail: kperrone@bsu.edu

P. Oliveira-Silva

University of Minho, Braga, Portugal

Ó. F. Gonçalves

Northeastern University, Boston, MA, USA
Results partially supported hypotheses and were discussed in light of existing knowledge based on empirical and theoretical sources.

Keywords Relationship satisfaction - Couples · Empathy · Conflict resolution · Physiological measures $\cdot$ Skin conductance Interbeat interval of the heart

Empathy towards one's romantic partner can be manifested physically, and physiological synchrony in heart rate and skin conductance between members of a couple has been linked to greater relationship satisfaction. The relationship between empathic processes and the central and peripheral activities have been demonstrated in recent research (e.g., Decety and Ickes 2009). When experiencing a close involvement with another person's mental states (e.g., recognizing, sharing, mirroring) individuals will automatically activate autonomic and somatic responses consistent with their interpretation of the other person's affective state. For example, Thomsen and Gilbert (1998) found that, during conflictual interactions, couples in which partners' patterns of heart rate and skin conductance were synchronous with one another had higher ratings of marital satisfaction.

One method of assessing physiological arousal involves examination of heart rate. In particular, the interbeat interval (IBI), represented by the temporal distance between successive $\mathrm{R}$ waves in the electrocardiographic curve, has been described as a sensitive physiological index not only to the level of arousal evoked by stimuli, but also to the valence and psychological processing of its context (Frazier et al. 2004). Another method of assessing physiological arousal is by examining skin conductance level 
(SCL). SCL is frequently used in psychophysiological research as an index of physiological arousal (Boucsein 2012). This electrodermal index has been largely used as an objective measure to assess sympathetic activity, providing an overall degree of autonomic arousal, conscious and unconscious emotional processing, and feeling states (Damasio 1994). SCL has been shown to change in response to stimulus significance, sustained attention, and affective intensity of a stimulus, reflecting the level of physiological arousability (Andreassi 2007; Cacioppo and Tassinary 1990).

Past research that has linked physiological arousal to poor conflict management (e.g., inability to manage conflict or tendency to avoid engaging in problem solving or conflict resolution strategies) and decreased relationship quality between partners in a romantic relationship (Levenson and Gottman 1985; Nealey-Moore et al. 2007). It makes sense that conflict management strategies such as avoidance or the inability to manage conflict through "giving in" or complying to the wishes of one's partner would be linked to higher arousal physiologically, since the motivation for such responses may come out of a "fear" reaction to one's unsettled partner. For example, research has linked cardiovascular reactivity during negative marital interactions to increased marital distress (e.g., NealeyMoore et al. 2007). Conversely, if physiological arousal and responsiveness are low, this could be indicative of a lack of engagement in the relationship or empathy with the partner (Andreassi 2007; Cacioppo and Tassinary 1990; Damasio 1994; Westbury et al. 2011). Thus, it is possible that physiological arousal could be linked to negative factors such as difficulty with conflict resolution, but also to positive factors such as empathy towards one's partner and relationship satisfaction. If couples are not able to exercise the appropriate amount of self-regulation of emotions and behaviors and subsequently experience too much or too little physiological arousal during disagreements or negative interactions, it may be more difficult for them to express empathy towards their partners or resolve conflicts with positive strategies. This can decrease relationship quality and may also have a negative effect on both mental and physical health (Smith et al. 2010).

The present research builds upon the extant literature as it assesses psychophysiological factors in relation to empathy, conflict resolution, and relationship satisfaction. In this study, we examined physiological reactivity along with self-reported empathy, conflict management, and relationship satisfaction in the context of emotionally laden interactions with romantic partners. Theoretical approaches to couples therapy often focus on empathy and conflict resolution to improve romantic relationship quality and satisfaction. For example, Integrative Behavioral Couple Therapy (IBCT; Christensen et al. 1995, 2004; Jacobson et al. 2000) emphasizes the role of empathy and conflict resolution as factors in romantic relationship satisfaction. We hypothesized that participants' level of empathy towards their partners would be closely related to physiological arousal, as measured by SCL and IBI, such that higher levels of empathy would be linked to an intermediate degree of higher physiological arousal and also higher relationship satisfaction. Further, we hypothesized that differences would be found in empathy and physiological arousal (as measured by SCL and IBI) based on the type of conflict resolution strategies used by participants. Finally, we hypothesized that differences would be found in empathy towards partner and relationship satisfaction based on the type of conflict resolution strategies used by participants.

\section{Method}

\section{Participants}

Participants were 31 students at a large Midwestern University (10 men, 21 women) who reported being in committed monogamous romantic relationship and attended data collection sessions with their partners. The partners were needed in order to create a realistic in vivo experience with participants. Participants' ages ranged from 19 to 25 years $(M=21.10 ; S D=1.47)$. Reported length of their romantic relationships were as follows: $32 \%$ between 3 months and 1 year; $29 \%$ had been together between 1 and 2 years; $11 \%$ between 2 and 3 years; $10 \%$ between 3 and 4 years; $10 \%$ between 4 and 5 years; $3 \%$ between 5 and 6 years; and $5 \%$ between 6 and 7 years. Eighty-seven percent of participants were in dating relationships, $7 \%$ were married, and $6 \%$ were engaged. Eighty-eight percent of participants had opposite sex partners and $12 \%$ of participants had same-sex partners. There were $65 \%$ Caucasian participants, $29 \%$ African American participants, $3 \%$ Asian American participants, and $3 \%$ multiracial participants.

\section{Procedures}

After receiving approval from the Institutional Review Board, the researchers recruited participants via the university research pools and via a recruitment email that was sent to all students in the university. Research credit was provided if applicable. No other incentives were offered. Participants were electronically mailed a consent form with a hyperlink. Once the participant gave consent to participate, he or she was directed to a survey by clicking on a hyperlink. Surveys consisted of demographic questions and scales measuring empathy, conflict resolution strategies, 
and relationship satisfaction. Within 1 week after completing online surveys, participants completed in-person data collection including measurement of SCL and IBI during an interactive task with the romantic partner. The in-person data collection occurred at the office of the primary researcher. For the in-person data collection portion of the study, participants and their partners were provided with an overview of the procedure, including information related with the psychophysiological devices and instructions about performing the interaction task. Participants and their partners were instructed to sit comfortably in two chairs facing one another and to interact with one another as naturally as possible. All subjects were instructed to abstain from alcohol, nicotine, and caffeine for at least $4 \mathrm{~h}$ before the data collection. All participants denied substance abuse disorders, cardiac diseases, use of psychotropic medication, or any other physical or mental condition that might affect the physiological measurements.

During the psychophysiological assessment, SCL and IBI were continuously recorded using the iWorx PK214 (iWorx SYSTEM, Inc., Dover, NH, USA) dual-channel biopotential amplifier designed for use on humans. SCL was analyzed through bipolar recording, using a pair of $\mathrm{Ag}$ / $\mathrm{AgCl}$ electrodes filled with $0.05 \mathrm{M}$ paste closely resembling sweat in its salinity, placed on the distal phalanges of the second and fourth fingers of the participants' nondominant hand. To ensure that neither dirt nor cosmetics would interfere with the measurement, the participants were asked to wash their hands with neutral soap and water immediately before the electrodes were placed. The Interbeat Interval (IBI) was chosen to monitor cardiac reactivity (rather than using heart rate to monitor cardiac reactivity) due to its more linear relationship with the changes in the parasympathetic and sympathetic autonomic branches (Berntson et al. 1995). Empirical evidence suggests that behaviorally relevant events are more strongly linked to the cardiac IBI than to the heart rate metric (Somsen et al. 2004). For an accurate ECG trace recording, a modified Lead II three-electrode montage was used, placing one electrode on each clavicle and another electrode slightly above the ankle of the left leg. The skin over the electrode placement areas was cleaned with alcohol and cotton to diminish the impedance of the skin and to improve the electrophysiological signal. The IBI values were automatically calculated by the LabScribe 2 software (iWorx Systems Inc., Dover, NH, USA) as the temporal interval (in milliseconds) between successive spikes, or R-waves, of the raw electrocardiogram (ECG), during the moments of interest. After sitting in a comfortable chair, participants were asked to keep their arms and legs as relaxed as possible, and to remove all jewelry from their wrists and ankles. After visual verification, the LabScribe 2 software (iWorx Systems Inc., Dover, NH, USA) was used to detect and correct artifacts due the presence of motion, and/or other type of artifacts, likewise to identify values outside the expected physiological range (i.e., SCL and IBI).

\section{Baseline Task}

The baseline task was designed to provide referential values of electrodermal and cardiac activities for all the sessions, holding verbal, social (i.e., the presence of their partner and the interaction between the members of the couple), and contextual (i.e., the clinical context and the use of the psychophysiological equipment) factors constant and at comparable levels within the segment. While recording SCL and IBI data, participants were presented with neutral valence and low-arousal set of full-color pictures selected from the International Affective Picture System database (IAPS; Lang et al. 1999, $n=18$ ). Each image was presented for $3 \mathrm{~s}$ (IAPS image numbers: 7004; 7052; 7059; 7150; 7161; 7175; 7179; 7185; 7186; 7187; $7188 ; 7205 ; 7235 ; 7236 ; 7238 ; 7248 ; 7245 ; 7820)$ separated by intervals lasting $2 \mathrm{~s}$, and participants were asked to name each image. All pictures were presented in the center of a 20-inch laptop screen during $90 \mathrm{~s}$, while the psychophysiological measures were recorded on a second laptop. SCL and IBI baseline values for each participant were calculated as the mean of SCL and IBI during the last $60 \mathrm{~s}$ of the baseline task. The first $30 \mathrm{~s}$ of the baseline were excluded to avoid the initial increase in physiological reactivity caused by anticipated anxiety associated with task performance. The SCL and IBI values during the interaction task were therefore computed by subtracting the mean value during each segment of interest of the baseline mean value (i.e., segment of interest minus baseline).

\section{Description of the Couples Interaction Task}

There were a series of 4 conditions with a problem focus and 4 conditions with a strengths focus. The 4 conditions in the problem-focused section of the study were as follows: For Condition 1, Partner 1 was instructed to speak for $2 \mathrm{~min}$ about problems or concerns related to the romantic relationship while Partner 2 listened without speaking. It should be noted that approximately half of the participants were designated as Partner I and spoke first, but the procedure was counterbalanced so that the other half of the participants were Partner 2 and spoke second. It was explained that only one partner was to talk at a time for each exercise. The listener was instructed to pay careful attention and advised that they would be asked to paraphrase what their partner said. For Condition 2, Partner 2 was given the next 2 min to communicate what he or she heard Partner 1 say without adding responses or reactions. For Condition 3, Partner 2 
was given an additional 2 min to either respond to the problem introduced by Partner 1, or to introduce a different problem or concern he or she wished to discuss. Partner 1 was instructed to listen carefully without speaking. For Condition 4, Partner 1 was instructed to take the next $2 \mathrm{~min}$ to communicate what he or she heard Partner 2 say. After the fourth condition, the partners were given $90 \mathrm{~s}$ of silence and instructed to think about positive aspects of their partner and their relationship.

The 4 conditions in the positive-focused section of the study (labeled conditions 5-8 here) were as follows: for Condition 5, Partner 1 was instructed to speak for $2 \mathrm{~min}$ about positive aspects of the partner and relationship (e.g., reasons for falling in love or being attracted to partner) while Partner 2 listened without speaking. For Condition 6, Partner 2 was instructed to speak for 2 min to communicate what he or she heard Partner 1 say without adding responses or reactions. For Condition 7, Partner 2 was given an additional 2 min to talk about positive aspects of Partner 1 while Partner 1 listened without speaking. For Condition 8, Partner 1 was instructed to take the next 2 min to communicate what he or she heard Partner 2 say.

After all conditions were completed, the equipment was removed and participants were provided with a debriefing form.

This task was informed by the theoretical framework used in Integrative Behavioral Couple Therapy (IBCT; Christensen et al. 1995, 2004; Jacobson et al. 2000). IBCT involves helping the couple improve communication about problem solving in the relationship (i.e., conflict resolution strategies). Couples are taught to listen to their partner and paraphrase what has been said because "...paraphrasing ensures that partners listen to each other and makes them more likely to see the other person's position" (Christensen et al. 1995, p. 54). This approach also encourages therapists to promote emotional acceptance between members of the couple through "...empathic joining around the problem" (Christensen et al. 1995, p. 54). Further, the IBCT approach was designed to include a broader range of couples than traditional approaches designed for heterosexual married couples, and thus it was applicable to the present study as we were open to including dating couples and same-sex couples as well as married and heterosexual couples.

\section{Instruments (Self Report)}

Empathy

Empathy was measured using the Interpersonal Reactivity Index (IRI; Davis 1983), adapted for couples by Péloquin and Lafontaine (Interpersonal Reactivity Index for Couples, IRIC 2010). The 13-item IRIC was designed to assess the degree of empathy individuals feel towards their romantic partner. Higher scores on the IRIC indicated higher levels of empathy. Participants responded on a 5-point, Likert-type scale $(0=$ Does not describe me well; $4=$ Describes me very well). Péloquin and Lafontaine (2010) reported adequate reliability for opposite-sex couples (alpha coefficients ranged from .74 to .84) and for same-sex couples (alpha coefficients ranged from .63 to .81). Convergent validity for the IRIC scales was demonstrated through significant correlations with other scales measuring general and dyadic empathy including the Self Dyadic Perspective-Taking Scale (SDPT; Long 1990; designed to measure perspective taking within the context of a specific relationship). Discriminant validity for the IRIC scales was demonstrated by weak associations with demographic variables that were theorized to be different from dyadic empathy (i.e., age, duration of relationship, and annual income; Péloquin and Lafontaine 2010). In addition, adequate test-retest reliability was found for the measure over a period of 18 months (Péloquin and Lafontaine 2010).

\section{Relational Conflict}

The Conflict Resolution Styles Inventory (CRSI; Kurdek 1994) was employed to measure individual's conflict management styles within their romantic relationships. The 16-item self rating of the CRSI was designed to measure four types of conflict resolution strategies: "Positive problem solving (e.g., focusing on the problem at hand), conflict engagement (e.g., exploding and getting out of control), withdrawal (e.g., reaching a limit and refusing to talk any further), and compliance (e.g., giving in with little attempt to present my side of the story)" (Kurdek 1994, p. 156). Higher scores indicate a higher frequency of engaging in these conflict styles during an argument with a significant partner. Participants responded on a 5-point Likert-type scale $(1=$ never to $5=$ always $)$. Internal consistency reliability for the four types of conflict management strategies were as follows: .89 (positive problem-solving), .89 (conflict engagement), .86 (withdrawal), and .88 (compliance; Kurdek 1994). Convergent validity was found though significant correlations between the CRSI and the Communication Patterns Questionnaire (Heavey et al. 1993; $p<.01$ ).

Relationship Satisfaction

Participants' relationship satisfaction was measured using the Relationship Assessment Scale (RAS; Hendrick et al. 1998). The 7-item RAS is a self-report global measure of relationship satisfaction. Participants responded on a 5-point Likert-type scale $(1=$ strongly disagree to $5=$ strongly agree). Higher scores indicated greater 
relationship satisfaction. Evidence of convergent validity was shown through significant correlations with the Dyadic Assessment Scale (Dinkel and Balck 2005). The RAS has demonstrated predictive validity for couples' relationship stability (Vaughn and Baier 1999).

\section{Results}

Prior to conducting the analyses to assess hypotheses, preliminary analyses were conducted to assess for effects of topic intensity and gender on the dependent variables. To assess for effects based on the degree of topic intensity discussed during the couples interaction tasks, we used the record of topics discussed by each participant that was kept during the data collection and asked 4 research team members to independently rate the intensity (high or low) of each topic discussed during the negative and the positive conditions. Independent ratings were examined for interrater reliability and responses were assigned to categories accordingly. In the event of a tie, a fifth rater would be asked to complete the ratings, but there were no ties on ratings of responses (i.e., either 3 out of 4 or 4 out of 4 raters agree on the valence of each topic). A $2 \times 2$ MANOVA was conducted with independent variables of negative condition topic intensity (low or high) by positive condition topic intensity (low or high) and dependent variables of interbeat interval of the heart (IBI), skin conductance level (SCL), and overall relationship satisfaction. Results indicated nonsignificant multivariate effects (Wilks' Lambda) for topic intensity in either condition. Oneway ANOVAs were used to examine potential gender differences in the dependent variables of IBI, SCL, and relationship satisfaction. No significant gender differences were found for gender and IBI, gender and SCL, or gender and relationship satisfaction.

We hypothesized that participants' level of empathy towards their partners would significantly effect physiological arousal, as measured by SCL and IBI, such that higher levels of empathy would be linked to physiological arousal and relationship satisfaction. This hypothesis was tested using a One-Way MANOVA to examine the effect of empathy towards the partner (high or low) on dependent variables IBI, SCL, and romantic relationship satisfaction. Multivariate analysis indicated overall significant effects [Wilks' Lambda $F(3,23)=6.24, p=.003$ ]. Thus, we proceeded to examine univariate effects among variables.

Results of univariate analyses indicated a nonsignificant effect for empathy on IBI or SCL. Results indicated a significant effect for empathy on relationship satisfaction $(F=14.74, p=.001)$. Examination of relationship satisfaction means revealed the mean for the high empathy group $(31.44, s d=3.19)$ was higher than the mean for the low empathy group $(25.50$, sd $=4.32)$. Thus, individuals with higher empathy towards their partners also had higher relationship satisfaction.

Next, we hypothesized that differences would be found in physiological arousal (as measured by SCL and IBI) based on the type of resolution strategy used by participants. A $2 \times 2 \times 2 \times 2$ MANOVA was used with dependent variables SCL and IBI and independent variables for each conflict resolution strategy (using high and low levels defined by a median split as categories for each): Positive Problem Solving, Conflict Engagement, Withdrawal, and Compliance. Wilks Lambda was nonsignificant for Positive Problem Solving, Conflict Engagement, and Withdrawal, indicating no significant effect for these strategies on IBI or SCL. A significant effect was found for Compliant conflict resolution strategies [Wilks Lambda $F(2,16)=7.83, p=.004]$. Examination of univariate effects revealed a nonsignificant effect for Compliance on SCL but a significant effect for Compliance on IBI $(F=13.89 ; p=.002)$. Examination of means revealed participants with high levels of Compliant conflict management strategies had higher physiological arousal as measured by IBI $(M=-.04, s d=.08)$ (lower IBI scores signify higher arousal because there is less time between heart beats) than individuals with lower levels of Compliant conflict management strategies $(M=-.02, s d=.07)$.

Finally, we hypothesized that differences would be found in empathy towards partner and relationship satisfaction based on the type of conflict resolution strategies used by participants. A $2 \times 2 \times 2 \times 2$ MANOVA was used with dependent variables empathy and relationship satisfaction and independent variables for each conflict resolution strategy (using high and low levels as categories for each): Positive Problem Solving, Conflict Engagement, Withdrawal, and Compliance. Wilks Lambda was nonsignificant for Conflict Engagement, Withdrawal, and Compliance, indicating no significant effect for these strategies on empathy and relationship satisfaction. A significant effect was found for Positive Problem Solving conflict resolution strategies [Wilks Lambda $F(2,20)=4.91$, $p=.01]$. Examination of univariate effects indicated significant effects for Positive Problem Solving on both empathy $(F=10.27, p=.004)$ and relationship satisfaction $(F=5.36, p=.031)$. Examination of means revealed individuals with high levels of Positive Problem Solving had higher levels of empathy towards their partner $(M=54.31, s d=.6 .29)$ than individuals with low levels of Positive Problem Solving $(M=45.00, s d=1.92)$. Additionally, individuals with higher levels of Positive Problem Solving had higher levels of relationship satisfaction $(M=31.07, s d=.3 .69)$ than individuals with low levels of Positive Problem Solving $(M=26.20$, $s d=1.92$.$) .$ 


\section{Discussion}

The use of physiological recording techniques to assess participants' level of arousal during an in vivo empathic interactive communication task with their partner was a strength of the study as it added a unique contribution to existing knowledge in this area. Further, much of the extant couples literature has focused on heterosexual or married couples, whereas this study added diversity by including both same-sex and opposite sex couples. The racial diversity of the sample was another strength of the study. Additionally, the findings of this study have a number of important implications for future practice.

Findings from this study demonstrated that individuals with higher empathy towards their partners also reported higher relationship satisfaction than individuals with lower empathy towards their partners. These findings are consistent with past research that has demonstrated the importance of empathy towards one's partner and romantic relationship satisfaction (e.g., Waldinger et al. 2004). Further, the findings from the present study indicated no significant differences based on gender or type of couple (same-sex vs. opposite sex couples).

Results from the present study indicated individuals with higher use of Positive Problem Solving conflict resolution strategies also had higher empathy towards their partners and higher relationship satisfaction. The finding that higher empathy towards one's partner is also linked to positive problem solving approaches makes intuitive sense, because when individuals are able to understand the other's feelings and viewpoints, it is easier to approach conflict in a more pro-social way. Further, this finding is consistent with past research that has linked positive problem solving strategies in couples to higher relationship satisfaction and quality (e.g., Bradbury and Karney 2004; Hanzal and Segrin 2009). The finding that higher empathy towards one's partner is also linked to positive problem solving approaches makes intuitive sense, because when individuals are able to understand the other's feelings and viewpoints, it is easier to approach conflict in a more pro-social way. That empathic accuracy may help to achieve a more solid understanding of the other's perspective before behaviorally reacting in a conflict situation, allowing the use of a more active and constructive problem solving strategy. An implication of this is the possibility that being more empathic towards their partners also facilitates the emergence of affective, cognitive, as well as motivational changes even when facing a hostile situation, before being overwhelmed with negative emotions, such as hurt, anger and frustration. Results from the present study indicated individuals who used compliant conflict resolution strategies had higher physiological arousal as assessed by IBI but not as assessed by SCL. No significant effects were found regarding physiological arousal for the other types of conflict resolution strategies. The Compliant conflict resolution style is described as a more passive although less damaging style, in which the person capitulates to their partner's desires and demands without attempting to assert or defend their position (Kurdek 1994). Items measuring this style include: "Not being willing to stick up for myself," "being too compliant," "not defending my position" and "giving in with little attempt to present my side of the issue." The increased physiological arousal could potentially be caused by the participant's acting in contrast to his or her wishes. For example, past research has shown a link between cognitive dissonance in induced compliance paradigms and physiological arousal (e.g., Croyle and Cooper 1983). Although participants might feel discouraged or frustrated when they are not able to assert their needs or desires in a more active way during relationship conflict, they tend to report and adopt the mindset that a partner should be more tolerant than they are able to truly be, when facing a real confrontational interaction. That is, they use compliance-based strategies but are not acting in a way that is congruent with their feelings and this could cause distress or increased physiological arousal. This may be a similar phenomenon to that research that has linked higher physiological arousal with lack of emotional expressiveness (Levenson and Gottman 1985; NealeyMoore et al. 2007; Smith et al. 2010).

Integrative Behavioral Couples Therapy (Christensen et al. 1995) emphasizes both change (e.g., replacing conflictual relationship behaviors with adaptive conflict resolution strategies) and acceptance (e.g., helping partners accept one another's limitations). In addition to facilitating behavior change, therapists can help partners develop greater understanding, acceptance, and empathy towards one another to help increase relationship satisfaction. Psychoeducation aimed at helping couples recognize how empathy looks and feels could be helpful. Perspectivetaking, can help each person feel heard and respected which could reduce defensiveness, withdrawal, and other unhealthy conflict management strategies. Psychoeducational presentations and experiential activities could disseminate specific information on perspective-taking, empathic concern, healthy communication styles, physiological arousal, and using adaptive conflict management strategies.

\section{Limitations of the Study and Directions for Future Research}

There were limitations in the present study that could be addressed in future research. One limitation was the 
relatively small sample size, although it should be noted that this type of research often utilizes smaller samples by necessity (i.e., due to the time involved in data collection on the part of the participants and researchers, and the need for the partners of participants to be present during the inperson data collection). Nonetheless, it is noted as a limitation that interpretations were made based on statistical analysis of mean differences in some groups that were very small. Generalizability of the sample was also limited in that all participants were college students. Therefore, it will be important to replicate this research in the future with a broader sample.

\section{References}

Andreassi, J. L. (2007). Psychophysiology: Human behavior and physiological response (5th ed.). Mahwah, NJ: Lawrence Erlbaum Associates.

Berntson, G. G., Cacioppo, J. T., \& Quigley, K. S. (1995). The metrics of cardiac chronotropism: Biometric perspectives. Psychophysiology, 32, 162-171.

Boucsein, W. (2012). Electrodermal activity (2nd ed.). New York, NY: Springer.

Bradbury, T. N., \& Karney, B. R. (2004). Understanding and altering the longitudinal course of marriage. Journal of Marriage and Family, 66, 862-879. doi:10.1111/j.0022-2445.2004.00059.x.

Cacioppo, J. T., \& Tassinary, L. G. (1990). Inferring psychological significance from physiological signals. American Psychologist, 45(1), 16-28. doi:10.1037/0003-066X.45.1.16.

Christensen, A., Atkins, D. C., Berns, S., Wheeler, J., Baucom, D. H., \& Simpson, L. E. (2004). Traditional versus integrative behavioral couple therapy for significantly and chronically distressed married couples. Journal of Consulting and Clinical Psychology, 72(2), 176-191. doi:10.1037/0022-006X.72.2.176.

Christensen, A., Jacobson, N. S., \& Babcock, J. C. (1995). Integrative behavioral couple therapy. In N. S. Jacobson \& A. S. Gurman (Eds.), Clinical handbook of couple therapy (pp. 31-64). New York, NY: Guilford Press.

Croyle, R. T., \& Cooper, J. (1983). Dissonance arousal: Physiological evidence. Journal of Personality and Social Psychology, 45, 782-791. doi:10.1037/0022-3514.45.4.782.

Damasio, A. R. (1994). The brain binds entities and events by multiregional activation from convergence zones. In H. H. Gutfreund \& G. G. Toulouse (Eds.), Biology and computation: A physicist's choice (pp. 749-758). River Edge, NJ: World Scientific Publishing Co.

Davis, M. H. (1983). Measuring individual differences in empathy: Evidence for a multidimensional approach. Journal of Personality and Social Psychology, 44(1), 113-126. doi:10.1037/00223514.44.1.113.

Decety, J., \& Ickes, W. (2009). The social neuroscience of empathy. Cambridge, MA: MIT Press.

Dinkel, A., \& Balck, F. (2005). An evaluation of the German relationship assessment scale. Swiss Journal Of Psychology, 64(4), 259-263. doi:10.1024/1421-0185.64.4.259.

Frazier, T. W., Strauss, M. E., \& Steinhauer, S. R. (2004). Respiratory sinus arrhythmia as an index of emotional response in young adults. Psychophysiology, 41(1), 75-83. doi:10.1046/j.14698986.2003.00131.x.
Hanzal, A., \& Segrin, C. (2009). The role of conflict resolution styles in mediating the relationship between enduring vulnerabilities and marital quality. Journal of Family Communication, 9(3), 150-169. doi:10.1080/15267430902945612.

Heavey, C. L., Layne, C., \& Christensen, A. (1993). Gender and conflict structure in marital interaction: A replication and extension. Journal of Consulting and Clinical Psychology, 61(1), 16-27. doi:10.1037/0022-006X.61.1.16.

Hendrick, S. S., Dicke, A., \& Hendrick, C. (1998). The relationship assessment scale. Journal of Social and Personal Relationships. 15(1), 137-142. doi:10.1177/0265407598151009.

Jacobson, N. S., Christensen, A., Prince, S. E., Cordova, J., \& Eldridge, K. (2000). Integrative behavioral couple therapy an acceptance-based, promising new treatment for couple discord. Journal of Consulting and Clinical Psychology, 68(2), 351-355. doi:10.1037/0022-006X.68.2.351.

Kurdek, L. A. (1994). Areas of conflict for gay, lesbian, and heterosexual couples: What couples argue about influences relationship satisfaction. Journal of Marriage and the Family, 56(4), 923-934.

Lang, P. J., Bradley, M. M., Cuthbert, B. N. (1999). International affective picture system: Instruction manual and affective ratings. Technical Report A-4. Gainsville, FL: The Center for Research in Psychophysiology, University of Florida.

Levenson, R. W., \& Gottman, J. M. (1985). Physiological and affective predictors of change in relationship satisfaction. Journal of Personality and Social Psychology, 49, 85-94. doi:10.1037/0022-3514.49.1.85.

Long, E. C. (1990). Measuring dyadic perspective taking: Two scales for assessing perspective taking in marriage and similar dyads. Educational and Psychological Measurement, 50(1), 91-103. doi:10.1177/0013164490501008.

Nealey-Moore, J. B., Smith, T. W., Uchino, B. N., Hawkins, M. W., \& Olson-Cerny, C. (2007). Cardiovascular reactivity during positive and negative marital interactions. Journal of Behavioral Medicine, 30, 505-519. doi:10.1007/s10865-007-9124-5.

Péloquin, K., \& Lafontaine, M. (2010). Measuring empathy in couples: Validity and reliability of the Interpersonal Reactivity Index for couples. Journal of Personality Assessment, 92, 146-157. doi:10.1080/00223890903510399.

Smith, T. W., Cribbet, M. R., Nealey-Moore, J. B., Uchino, B. N., Williams, P. G., MacKenzie, J., et al. (2010). Matters of the variable heart: Respiratory sinus arrhythmia response to marital interaction and associations with marital quality. Journal of Personality and Social Psychology, 100, 103-119. doi:10.1037/a0021136.

Somsen, R. M., Jennings, J., \& van der Molen, M. W. (2004). The cardiac cycle time effect revisited: Temporal dynamics of the central-vagal modulation of heart rate in human reaction time tasks. Psychophysiology, 41(6), 941-953. doi:10.1111/j.14698986.2004.00241.x.

Thomsen, D. G., \& Gilbert, D. G. (1998). Factors characterizing marital conflict states and traits: Physiological, affective, behavioral and neurotic variable contributions to marital conflict and satisfaction. Personality and Individual Differences, 25(5), 833-855. doi:10.1016/S0191-8869(98)00064-6.

Vaughn, M. J., \& Baier, M. E. (1999). Reliability and validity of the relationship assessment scale. American Journal of Family Therapy, 27(2), 137-147. doi:10.1080/019261899262023.

Waldinger, R. J., Schuz, M. S., Hauser, S. T., Allen, J. P., \& Crowell, J. A. (2004). Reading others' emotions: The role of intuitive judgments in predicting marital satisfaction, quality, and stability. Journal of Family Psychology, 18(1), 58-71. doi:10.1037/ 0893-0893-3200.18.1.58.

Westbury, R., Neumann, D., \& Waters, A. (2011). Extending empathy research towards animals. In Psychology of empathy. Hauppauge, NY: Nova Science Publishers Inc. 